\title{
Density models for streamer discharges: Beyond cylindrical symmetry and homogeneous media
}

\author{
A. Luque ${ }^{\mathrm{a}, *}, \mathrm{U}$. Ebert ${ }^{\mathrm{b}, \mathrm{c}}$ \\ ${ }^{a}$ IAA-CSIC, P.O. Box 3004, 18080 Granada, Spain \\ ${ }^{\mathrm{b}}$ Centrum Wiskunde E' Informatica (CWI), Amsterdam, The Netherlands \\ ${ }^{\mathrm{c}}$ Department of Applied Physics, Technische Universiteit Eindhoven, The Netherlands
}

\section{A R T I C L E I N F O}

\section{Article history:}

Available online 21 April 2011

\section{Keywords:}

Streamers

Electric discharges

Density models

\begin{abstract}
A B S T R A C T
Streamer electrical discharges are often investigated with computer simulations of density models (also called reaction-drift-diffusion models). We review these models, detailing their physical foundations, their range of validity and the most relevant numerical algorithms employed in solving them. We focus particularly on schemes of adaptive refinement, used to resolve the multiple length scales in a streamer discharge without a high computational cost. We then report recent results from these models, emphasizing developments that go beyond cylindrically symmetrical streamers propagating in homogeneous media. These include interacting streamers, branching streamers and sprite streamers in inhomogeneous media.
\end{abstract}

(c) 2011 Elsevier Inc. All rights reserved.

\section{Introduction}

Streamers [1-4] are transient electrical discharges that appear when a non-conducting medium is suddenly exposed to a high electric field, often localized around a high-potential pointed electrode. While the average background field might be too low for plasma generation by electron impact on neutral molecules, the streamer discharge channel can enhance the electric field at its growing tip so strongly that it can create additional plasma and propagate nevertheless. The streamer achieves this through a very nonlinear dynamics with an intricate inner structure, locally very steep density gradients and space charge concentrated in very thin layers. This structure generates the local field enhancement and maintains propagation. Therefore, the accurate simulation of single, cylindrically symmetric streamer channels is far from trivial, even if the charged species are approximated by their densities as is usually done.

It should be noted that the full process evolves on even more scales. On the one hand, when the electric field ahead of the streamer head is very high, single electrons can run away and generate hard radiation through Bremsstrahlung. To describe these effects, single electrons have to be followed in a particle model; numerical methods to track these particles in an efficient manner and the derivation of density models are discussed in this special issue by Li et al. [5]. On the other hand, in the laboratory, in technological applications and in lightning related processes, hundreds to ten-thousands of streamer channels can propagate next to each other. In this case it is vital to develop models at a more macroscopic scale than the density approximation. Through model reduction, one can derive moving boundary models [6-10] for the underlying ionization fronts, or one even can try to develop models for the streamer channel as a whole.

Within the current short review, we discuss the justification for density models for streamers, the numerical solution strategy, and then some results that go beyond single cylindrically symmetric streamers in homogeneous media. We treat interacting streamers, first through the trick of considering an infinite array of identical streamers next to each other, then

\footnotetext{
* Corresponding author.

E-mail addresses: luque@iaa.es, aluque@iaa.es (A. Luque).
} 
through full 3D simulations. Next, we discuss streamer branching in full 3D. Finally we discuss how to simulate the emergence of sprite streamers from the Earth's ionosphere; a peculiar issue is here how to combine the very different scales of the electric fields generated by the thundercloud-ground-ionosphere system with the fine inner structure of the discharge and with the density of the atmosphere, that varies over a length-scale of about $7 \mathrm{~km}$.

With the density models, here we focus on the oldest and most extensively investigated family of numerical streamer models; they are also known as fluid models, continuous models or reaction-drift-diffusion models.

\section{Model formulation}

\subsection{The density model}

A classical density model for a streamer discharge has the structure of a reaction-drift-diffusion equation for the electrons and reaction equations for various ions and excited species coupled to the electric field

$$
\begin{aligned}
& \frac{\partial n_{e}}{\partial t}=\nabla \cdot\left(n_{e} \mu_{e} \mathbf{E}\right)+\nabla \cdot D_{e} \nabla n_{e}+S_{e}^{i m}+S_{e}^{p h}, \\
& \frac{\partial\left[Z_{i}\right]}{\partial t}=S_{i}^{i m}+S_{i}^{p h}, \quad i=1, \ldots, N, \\
& \epsilon_{0} \nabla \cdot \mathbf{E}=q, \quad \mathbf{E}=-\nabla \phi .
\end{aligned}
$$

Here $n_{e}$ is the electron number density and $\left[Z_{i}\right]$ is the density of the heavy species $Z_{i}$ denoting a positive or negative ion or an excited state, and $\mu_{e}$ and $D_{e}$ are mobility and diffusion coefficients of the electrons. $S_{e, i}^{i m}$ denote mostly local generation or loss of species due to reactions at direct encounter of particles; the most prominent example is electron impact ionization, i.e., the generation of electron-ion pairs through impact of electrons on neutrals; the efficiency of this process strongly depends on the electron energy that in turn is determined by gas density and electric field. $S_{e, i}^{p h}$ denote the generation of the species through radiative transport in a generically nonlocal process; the most prominent example in streamer physics is the generation of electron- $\mathrm{O}_{2}^{-}$pairs through photo-ionization in air. Finally, $\mathbf{E}$ is the electric field, $\phi$ the electric potential, and $q$ is the local space charge, determined as $q=\sum_{i} q_{i}\left[Z_{i}\right]-\mathrm{e} n_{e}$; here e is the elementary charge and $q_{i}=-\mathrm{e}, 0$, +e is the charge of species $Z_{i}$.

\subsection{The underlying approximations}

We list here the main approximations underlying this model. For a more thorough derivation of the model, we refer to $[5,11,12]$ and references therein.

1. The electrons are much more mobile than the ions, and the dynamics takes place on their time scale, therefore ion motion is neglected-the validity of this approximation often has to be checked after the longest relevant time scales are found through a simulation. This approximation saves computing time and removes unnecessary complexity but it is not essential: ion mobility can be easily incorporated in streamer numerical models.

2. The electron motion is approximated by drift and diffusion within the local field. This entails that the electrons rapidly relax to an equilibrium velocity distribution where the acceleration by the electric field exactly cancels the momentum losses due to collisions with other particles, and that spatial or temporal variations of the electric field are not important. The accuracy of the drift-diffusion approximation and the local field approximation for electron currents in streamer ionization fronts was verified in [12] for electric fields up to some threshold; this statement holds for arbitrary gas density once the threshold electric field is rescaled accordingly. However, when the field at the streamer head exceeds a limit $(180 \mathrm{kV} / \mathrm{cm}$ in air at standard temperature and pressure (STP) $[5,11,13,14])$, some electrons in the tail of the electron energy distribution function might not relax to a steady velocity anymore, but run away with a field dependent probability. Above the thermal runaway electric field $(260 \mathrm{kV} / \mathrm{cm}$ in STP air [15-17]) the bulk of the electrons will keep accelerating up to relativistic energies and the drift approximation breaks down.

3. The field is calculated with electrostatic approximation. For typical current densities and diameters of streamers [18], this approximation is justified.

4. The ensembles of electrons and ions can be treated in density approximation. The validity of this approximation depends first on the gas density, and second on the specific region within the streamer. First, streamers in different gas densities $n$ are related through similarity relations or Townsend scaling in very good approximation $[18,19]$; these relations imply that the electron density $n_{e}$ in a streamer scales as $n^{2}$, and that the intrinsic length scales scale as $1 / n$. Consequently, the total number of electrons in similar parts of streamers scales as $1 / n$. This implies that the density approximation in all regions of the streamer becomes better when the gas density decreases. On the contrary, the density approximation for electrons and ions becomes worse when the density of the neutral medium increases, and it has to be reconsidered at the high densities of liquids. ${ }^{1} \mathrm{~A}$ review of validity and corrections to these similarity solutions is given in [18].

\footnotetext{
${ }^{1}$ Other approximations to be reconsidered in streamers at liquid densities are the absence of heating and the neglect of electron-electron and electron-ion collisions in the source terms $S^{i m}$. In gases, only electron-neutral collisions are included in typical streamer models, but the degree of ionization and therefore the relative frequency of electron-electron or electron-ion collisions increases with increasing density because $n_{e} / n \propto n$.
} 
Second, different spatial regions of the streamer have to be distinguished: interior, front and outer region. The streamer interior has large densities of electrons and ions and little inner structure; the density approximation is certainly justified there. In the outer region, electron densities are typically very low (except for high discharge repetition frequencies or with other strong ionization sources) and densities should rather be interpreted as probabilities; however, a change of this low background ionization has essentially no influence on streamer propagation in typical situations [20], but it can have an influence on branching and "feather" formation in very pure gases with little nonlocal photo-ionization [21,20]. Finally, the streamer ionization front connects interior and exterior and the electron density can develop steep gradients, in particular, in high electric fields and with little or no nonlocal photo-ionization [12,20-22]; in these extreme cases, the electron density can vary by a factor $\sim 10^{10}$ in a few tenths of a millimeter at atmospheric pressure [20]; for analytical approximations of the density gradients, we refer to the review [23]. Within this region, the local density approximation can break down as electrons with a higher energy are ahead of others. The electron energies are then determined not only by the local electric field, but also by the gradient of the electron density; this effect can be incorporated in a modification of the impact ionization term; or alternatively, the model can be extended by an electron energy equation [11]. These terms can be included in the general structure of the reaction-drift-diffusion model above and do not require any separate numerical strategy. According to [11], the density approximation describes streamer propagation well, as long as no massive electron runaway sets in. However, to properly describe the effect of density fluctuations and electron run-away on streamer stability, a particle approach should be chosen. Presently available models are Monte Carlo particle models $[16,24,25,14]$ and spatially hybrid models [11,5] that combine particle and density models in different volumes of the simulation.

\subsection{Typical models in air}

The general structure of the density model defined above has been recognized decades ago but the search for better reaction and transport parameters in different gases goes on. Most modeling work is devoted to air modeled as a mixture of nitrogen and oxygen.

The impact ionization term $S^{i m}$ in air includes the generation of free electrons and positive ions through electron impact on neutral molecules as well as electron losses due to attachment to oxygen. In particular the first reaction strongly depends on the electric field. There is also field dependent detachment of electrons from $\mathrm{O}_{2}^{-}$[26], however, it is typically neglected in air, and it indeed only contributes in a relevant manner in nitrogen with admixtures of oxygen as low as 1 p.p.m., but not at the $20 \%$ oxygen content in air [20].

This is because air has very strong nonlocal photo-ionization-for a historical review of this concept, we refer to the introduction of [21]. In oxygen-nitrogen mixtures such as air, photo-ionization is in most cases included through the Zhelezniak model [27]. In this model, the states $\mathrm{b}^{1} \Pi_{\mathrm{u}}, \mathrm{b}^{1}{ }^{1} \Sigma_{\mathrm{u}}{ }^{+}$and $\mathrm{c}_{4}^{\prime 1} \Sigma_{\mathrm{u}}^{+}$of molecular nitrogen, populated by impact excitation in the high-field regions of the streamer, decay by emitting photons, some with energies above the ionization threshold of molecular oxygen, $12.1 \mathrm{eV}(1025 \AA)$. The Zhelezniak model assumes that the excitation of the emitting states is roughly proportional to the impact ionization. Then the production of electron-ion pairs per unit of volume and time is written as

$$
S_{p h}(\mathbf{r})=\frac{\xi}{4 \pi} \frac{p_{q}}{p+p_{q}} \cdot \int \frac{h\left(p\left|\mathbf{r}-\mathbf{r}^{\prime}\right|\right) S_{e}^{i m}\left(\mathbf{r}^{\prime}\right) d^{3}\left(p \mathbf{r}^{\prime}\right)}{\left|p \mathbf{r}-p \mathbf{r}^{\prime}\right|^{2}},
$$

where $\xi$ is a proportionality constant, $h$ is the absorption function of photo-ionizing radiation and $p_{q}=60$ torr $=80 \mathrm{mbar}$ is the quenching pressure of the emitting states. There is some uncertainty in this value: we use the value given by Legler [28]; other authors $[27,29-31]$ use $p_{q}=30$ torr. Photo-ionization might also be very strong in hydrogen-helium mixtures that dominate the atmospheres of gas giants like Jupiter and Saturn [32]; however, quantitative models are not available for this mixture.

The reaction rates strongly depend on electron energies (that are typically parameterized by the electric field that accelerates the electrons), but also the transport coefficients $\mu_{e}$ and $D_{e}$ depend on it, though weakly. In most literature, they are treated as constant. Furthermore, in general the diffusion coefficient $D_{e}$ is a tensor with different components parallel and perpendicular to the electric field [5], but most investigators approximate it as a scalar. Reaction and transport coefficients are mostly calculated in advance from cross-section data such as [33] by solving the Boltzmann equation for electrons with numerical codes such as BOLSIG+ [34]. Alternatively, they also can be calculated by performing Monte Carlo simulations with good statistics [5,11]. Much work is currently devoted to improvements of the cross-section data as well as to the evaluation of the Boltzmann equation [35].

On the other hand, it can be stated that the propagation of streamer discharges seems to be amazingly robust both against model changes in simulations [20] accounting for different attachment, photo-ionization or background ionization rates; this is in agreement with experiments where streamer velocities are quite robust against changes of gas composition [21,32]. The reason is probably the strongly nonlinear dynamics of the streamer that approaches generic attractors of the dynamics independently of microscopic details. E.g., in streamers in nitrogen with 1 p.p.m. oxygen, the effect of background ionization or photo-ionization can hardly be distinguished, and major changes of the photo-ionization model in air have only minor effects on streamer velocity [20]. 


\section{Numerical schemes}

Generally speaking, the physical models that underlie numerical streamer simulations are always small variations on the model discussed above. These variations include e.g. the use of field-dependent mobility and diffusion and the inclusion of kinetic equations for additional chemical reactions.

To reduce computational demand, almost all streamer simulations are performed in cylindrical symmetry with radial and longitudinal coordinates $(r, z)$ although below we will discuss some recent extensions to real $3 \mathrm{~d}$.

\subsection{Discretization of the transport equation}

The main challenge in the numerical solution of the densities of charged particles in streamers arises from the very steep gradients in the streamer tip. The choice of discretizations has been largely driven by the requirement of handling these gradients efficiently. The first studies [36-39] used variations on the Flux-Corrected Transport (FCT) algorithm developed by Zalesak [40], combined with a modified Euler scheme for the time integration. In 1995 Kulikovsky [41] introduced a scheme based on improvements of the Scharfetter-Gummel algorithm that has subsequently been used also by Liu and Pasko [29] and Bourdon et al. [42]. On the other hand, first-order upwind schemes were used in [43] but they seemed to be too diffusive, and they over-stabilized the streamer fronts. In [22], an upwind-biased scheme was applied that used the Koren flux limiter [44] to switch between first-order upwind and third order upwind-biased.

These discretizations may be combined with various algorithms to work with different resolutions in different volumes of the domain. The importance of this part of the numerical scheme stems from the multi-scale nature of the streamer process discussed above. Kulikovsky [31] used a "window" with a fixed, higher resolution that followed the streamer head, while Pancheshnyi et al. [45], Bessières et al. [46] and Eichwald et al. [47] used a rectangular product grid with smoothly varying space steps. Min et al. [48], Nikandrov et al. [49] and Pancheshnyi et al. [50] have used dynamically adaptive meshes.

Montijn et al. [22] used a scheme of nested uniform grids generated adaptively with a criterium based on the second derivatives of the species and charge densities as follows. The refinement builds a hierarchy of grids starting from the coarsest grid that covers the entire volume and descending to finer grids. To decide which areas to refine, at each grid at level $l$, monitor functions $M_{u}$ are calculated, where $u$ stands for the electron density or space charge density. These functions come from the discretization of

$$
M_{u}(r, z)=\left(\Delta r^{l}\right)^{2}\left|\frac{1}{r} \frac{\partial}{\partial r}\left(r \frac{\partial u}{\partial r}\right)\right|+\left(\Delta z^{l}\right)^{2}\left|\frac{\partial^{2} u}{\partial z^{2}}\right|,
$$

where $\Delta r^{l}$ and $\Delta z^{l}$ are, respectively, the $l$-level grid resolutions in $r$ and $z$. The refinement criterium is then specified from grid-independent refinement tolerances $\epsilon_{u}$ as

$$
\text { refine all grid cells } i, j \text { where } \frac{M_{u}\left(r_{i}^{l}, z_{j}^{l}\right)}{\max u_{i j}^{l}} \geqslant \epsilon_{u} \text {. }
$$

Streamer fronts are pulled, i.e. the dynamics is determined in the leading edge of the ionization front [51]. For curved fronts as occurring in streamers, the leading edge is limited by the decay of the electric field. Therefore the refinement criterium (4) is often insufficient in the leading edge of the front. The approach used in [22] is to use a region larger than given by (4) and extend all grids in the leading edge up to the layer where the electron density is below a small threshold. However, when photo-ionization is included in the simulations the electron density ahead of the front decays much slower and this approach leads to an overextension of the refinement grids. Instead one can use a criterium based on the absolute value of the electric field, adding to (4) the additional criterium

$$
\text { refine grid cells } i, j \text { where }\left|E_{i j}\right| \geqslant E_{\text {threshold }},
$$

with an appropriately chosen $E_{\text {threshold }}$.

\subsection{Strategies for the Poisson-equation}

Solving the Poisson equation is usually one of the most expensive parts of simulations of density models for streamers. It is challenging, because the solution is nonlocal and instantaneous, i.e., the boundary conditions together with the very localized charge distribution in the simulation domain determine the electric field instantaneously. The Poisson equation is most often discretized in a Cartesian grid by a five-point second order scheme. The resulting linear system is then solved using either iterative methods, usually from the Successive Over-Relaxation (SOR) family $[29,47,52,53]$ or by direct methods such as cyclic reduction [22,54] and SuperLU [55]. In general, direct methods can be much faster than iterative methods for these problems, but they perform similarly if the iterative method is provided with a good initial guess from the solution of a previous time step. 
Even with fast algorithms, solving the Poisson equation is a major computational bottleneck for streamer simulations in full three dimensions. Two earlier 3D streamer simulations [56,26] constitute a proof of principle that such computations can be performed. An efficient and parallelizable approach for that problem is described in [57]; it extends the grid refinement technique from [22] to 3D simulations. This method is efficient for streamer dynamics that can be represented in cylindrical coordinates $(r, z, \theta)$ with a relatively low resolution in the axial coordinate $\Delta \theta=2 \pi / N$.

The discrete Fourier transform (DFT) in $\theta$ of the electrostatic potential $\phi(r, z, \theta)$ reads

$$
\tilde{\phi}_{k}(r, z)=\sum_{n=0}^{N-1} \phi(r, z, 2 \pi n / N) e^{-2 \pi i k n / N},
$$

which implies the usual properties of the DFT of a real quantity with $N$ even,

$$
\tilde{\phi}_{k}(r, z)=\tilde{\phi}_{N-k}^{\star}(r, z), \quad \tilde{\phi}_{0}(r, z)=\tilde{\phi}_{0}^{\star}(r, z), \quad \tilde{\phi}_{N / 2}(r, z)=\tilde{\phi}_{N / 2}^{\star}(r, z),
$$

where ${ }^{\star}$ indicates complex conjugation. The benefit of working in Fourier space is that different modes $k$ are decoupled and can be solved in parallel. The Laplace operator $\nabla^{2}$ can be decomposed into $\nabla^{2}=\nabla_{r z}^{2}+\frac{1}{r^{2}} \frac{\partial^{2}}{\partial \theta^{2}}$ and the midpoint discretization of the second derivative with respect to $\theta$ is

$$
D_{\theta}^{2} \phi_{j}=\frac{\phi_{j-1}-2 \phi_{j}+\phi_{j+1}}{\Delta \theta^{2}}
$$

where for clarity we have omitted the indices for $r$ and $z$. Applying the DFT as defined in (6) we obtain

$$
D_{\theta}^{2} \tilde{\phi}_{k}=\frac{\tilde{\phi}_{k} e^{-2 \pi i k / N}-2 \tilde{\phi}_{k}+\tilde{\phi}_{k} e^{2 \pi i k / N}}{\Delta \theta^{2}}=-\left|w_{k}\right|^{2} \tilde{\phi}_{k},
$$

where

$$
\left|w_{k}\right|^{2}=\frac{2}{\Delta \theta^{2}}(1-\cos k \Delta \theta)
$$

Note that as $\Delta \theta \rightarrow 0,\left|w_{k}\right|^{2} \rightarrow k^{2}$, giving the well known form of the continuous Fourier transform of a second derivative.

Finally, the equation for $\tilde{\phi}$ including the $r, z$ terms is a two-dimensional Helmholtz equation

$$
\left[\nabla_{r z}^{2}\right] \tilde{\phi}_{k}(r, z)-\frac{\left|w_{k}\right|^{2}}{r^{2}} \tilde{\phi}_{k}(r, z)=-\tilde{q}_{k}(r, z) / \epsilon_{0}
$$

where $\left[\nabla_{r z}^{2}\right]$ is the discrete Laplace operator in $(r, z)$ and $\tilde{q}_{k}(r, z)$ is the DFT of the space charge density. The available numerical methods for this equation are basically the same as for the Poisson equation (where the second term on the left hand side is missing) but (11) makes it possible to solve the equations independently and hence in parallel for each $k$.

Unfortunately, the reaction terms in (1a) and (1b) are nonlinear so the reaction-drift-diffusion part of the model must be solved in real space, where it can also be trivially parallelized. Hence an efficient parallel algorithm must switch between Fourier and real space at each time step. This is relatively fast by using widely available Fast Fourier Transform (FFT) codes.

Since the Poisson equation is usually solved in a Cartesian grid, a second problem concerns the modeling of a curvilinear or point-shaped electrode. This is an important aspect of streamer simulations since in experiments streamers almost always emerge from a pointed electrode. One common approach, introduced by Babaeva and Naidis [58], is to consider a spherical needle electrode where the computational domain of the densities begins only at the tip of the electrode, and to calculate its influence by the method of image charges. Another approach was proposed in [59], based on a simplified version of the method of virtual charges. A similar approach that does include the needle electrode into the domain of density computations is the Charge Simulation Method [60,61] that was implemented for streamers in Section 7.2 of [62]. Recently the use of immersed boundaries was introduced in streamer simulations by Celestin et al. [55].

A non-planar electrode can be also simulated by using curvilinear coordinates, adapted to the geometrical shape of the electrodes [63,64]; this is particularly appropriate if the pointed-electrode has a parabolic shape.

\subsection{Strategies for the nonlocal photo-ionization}

In the first simulations of streamers, photo-ionization was not included in the model but rather substituted by an unrealistically high constant pre-ionization [36]. Later, the Zhelezniak model was introduced by directly evaluating the integral (2), i.e., by summing the effect of each emitting source on each grid point in the simulation domain. The number of computations needed in this approach scales quadratically with the total number of grid points, growing much faster than the number of computations required for the Poisson equation or the advection-diffusion time stepping. One can use a coarser grid for photo-ionization calculations [31], but this seems to introduce spurious effects, resulting in a hollow streamer channel. 
Another approach, used in [65], is to approximate the spatial distribution of the photo-ionizing emitters by a sphere centered at the center of mass of radiation and with a radius equal to some characteristic radius of the streamer.

A more accurate approach is to approximate the Zhelezniak integral by the solution of elliptic partial differential equations. This method was introduced independently by Segur et al. [66] and Luque et al. [67], and further refined in [42,68,69].

\section{Beyond the cylindrically symmetrical streamer in a homogeneous gas}

The keystone of streamer simulations consists of a single cylindrically symmetrical streamer propagating in a homogeneous medium. As we mentioned above, this is by itself a physically relevant and challenging numerical problem. Nevertheless, in recent years several extensions to this paradigm have been introduced that investigate a wider range of problems. Here we review three of them: the interaction between neighboring streamers, the branching of a streamer channel and the propagation of streamers in the upper atmosphere, with a significant gradient of air density.

\subsection{Streamer interaction}

Streamers very rarely appear in isolation; most often they belong to bunches of many streamers, either because they have emerged at different points of a wire electrode or because they are contained in a highly branched tree emerging from a point electrode. Since each streamer carries charge, they interact electrostatically.

\subsubsection{Bunches of streamers and Saffman-Taylor streamers}

The first work to address the interaction of neighboring streamers was done by Naidis [70]. He used a quasi-2D density model to estimate the effect of the interaction with neighboring streamers in a regular one-dimensional lattice of well-separated streamers. The underlying assumption was that the radius of each streamer is much smaller than the distance to the closest neighbor.

A further step was demonstrated in [71], where the transversal dynamics of interacting streamers is taken into account by a modification of a single-streamer model. As shown in Fig. 1 (left) the interaction between neighboring streamers is incorporated as a lateral homogeneous Neumann boundary condition of the electrostatic potential. As a first step, Ref. [71] was limited to a 2D (planar) model of negative streamers without photoionization. However the technique can also be applied to more realistic situations [72].

The outcome, after a transient regime where each streamer expands, is a steady state where each channel fills half of the available width $L$ (the distance between one streamer and its neighbor). In this steady state, the electric field far behind the array of streamer heads (at a distance of a few times $L$ ) is completely screened. The numerical solution shows a remarkable agreement with the Saffman-Taylor analytical solution of the well-known problem of viscous fingering in Hele-Shaw cells (Fig. 1, right) $[73,74]$.

The analogy between streamers and viscous fingering is explicit in moving boundary models [6,8-10,75]; for a detailed recent review of such models, we refer to [23].

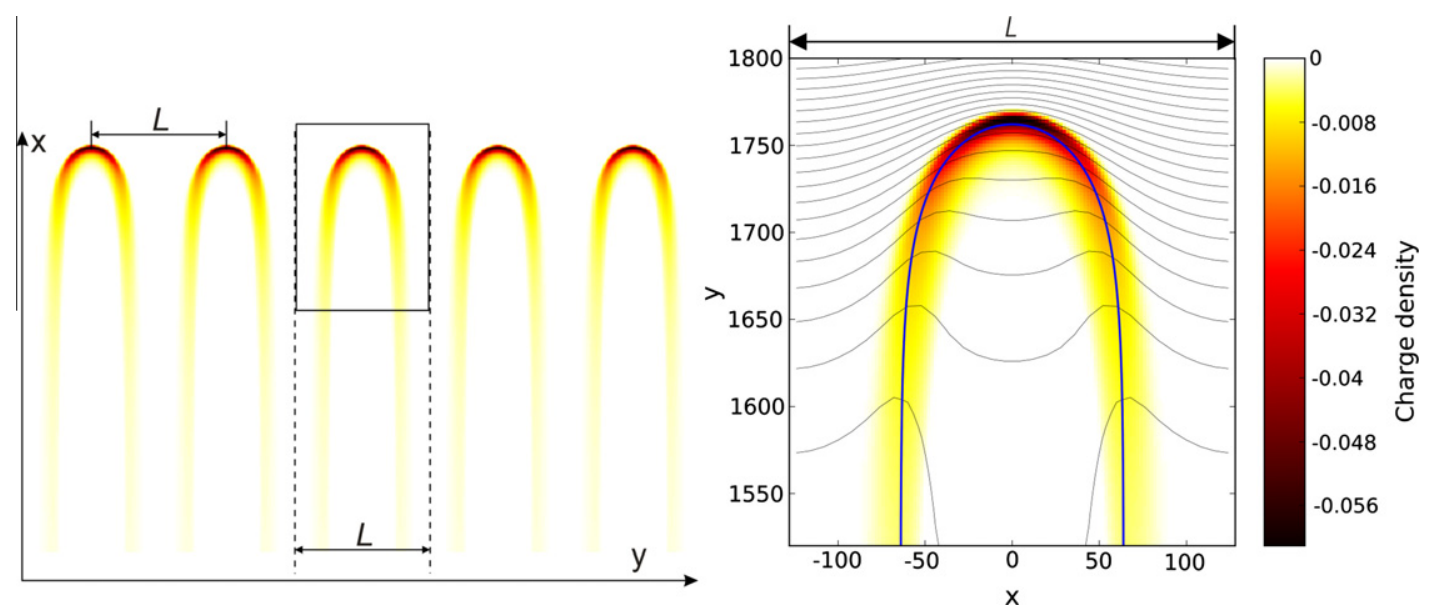

Fig. 1. Left: periodic array of negative streamers. The interaction between neighboring streamers can be incorporated into a density model by imposing homogeneous Neumann boundary conditions on the electrostatic potential on the dashed lines. Right: The resulting streamer profile fits the analytical solution of a selected Saffman-Taylor finger, with width $L / 2$. Reproduced from [71] (Copyright 2008 by the American Physical Society). 

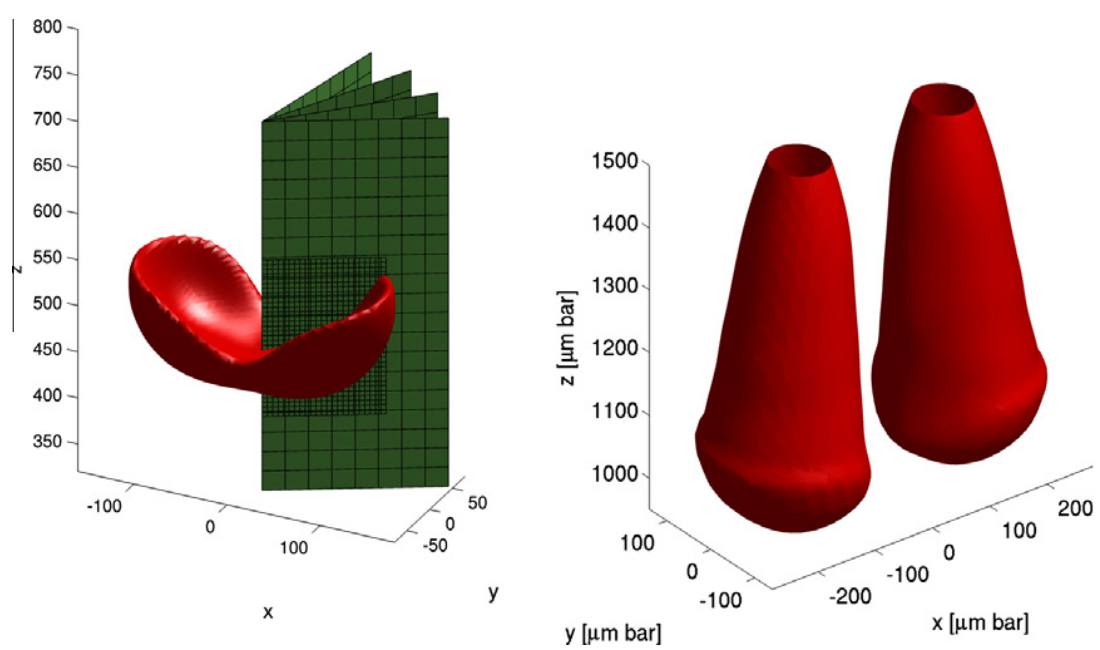

Fig. 2. Left: Scheme of refinement for the $3 \mathrm{~d}$ simulation approach described in the text. The red surfaces surround the streamer head, defined by its charge density. The slices represent the numerical resolution of the density equations: they are equi-spaced in the angular direction but in the $r$, $z$ plane they contain a scheme of nested grids, common for all slices. Right: Surfaces of constant electron density of two interacting negative streamers in nitrogen at atmospheric pressure propagating downwards in a homogeneous electric field of $80 \mathrm{kV} / \mathrm{cm}$. Reproduced from [57] (Copyright 2008 by the American Physical Society). (For interpretation of the references to colour in this figure legend, the reader is referred to the web version of this article.)

\subsubsection{Interaction between two streamer heads}

Another approach to the problem of streamer interaction was presented in [57], where the method of 3d calculations explained in Section 3.2 was used to study the interaction between two negative streamer heads propagating in parallel in a homogeneous electric field (see Fig. 2).

In general, for a given separation between the streamer heads, the outcome of the interaction depends on the gas composition. In cases where photo-ionization is absent such as in pure nitrogen (as far as it can be realized in the laboratory [21]), the electrostatic repulsion between the charges in the streamer heads drives them apart, bending the streamer channels outwards. However, for gas compositions where photo-ionization plays an important role, such as air, the ionization in the volume between the two heads counteracts the electrostatic repulsion between them, driving the two streamers to merge. In general the outcome of the streamer interaction would therefore depend on the separation between them and the balance of these two counteracting processes.

Until now, simulations of streamer interaction have been limited to relatively short times and simple configurations. A strong limitation is the need for 3D simulations, still expensive computationally. Likely, more realistic studies of streamer interaction will rely on model reduction to abstract from the details of each single streamer and to focus instead on the behavior of a streamer ensemble.

\subsection{Branching}

The branching of a streamer channel is perhaps the most challenging phenomenon for numerical simulations. The main reason is that branching is caused by a dynamical instability at the streamer front, where small irregularities in the very thin space-charge layer grow and eventually form new streamers. Therefore simulations of the branching process demand a high numerical accuracy to rule out numerical artifacts. This difficulty is compounded by the fact that branching completely breaks the cylindrical symmetry of a single streamer channel, thus requiring full 3D simulations for the complete understanding of the phenomenon.

In 2002 Arrayás et al. [76] studied the branching of a negative streamer in a minimal model that did not include photoionization. There, cylindrical symmetry was imposed and the result was interpreted as an upper bound for branching. The resulting picture was one of branching due to a Laplacian instability, in close analogy to other well studied physical models such as diffusion-limited aggregation and viscous fingering. Following this result, the numerical scheme was improved by Montijn et al. [22], who showed that branching time converged as the highest resolution of the numerical grid was improved [77], thus ruling out that branching was due to numerical artifacts.

Although those results imposed a cylindrical symmetry on the streamer, we can show now that they are robust when that restriction is removed. We use the method of 3D simulations detailed in [57] to investigate the dynamics of a negative streamer without photo-ionization under small perturbations that break its cylindrical symmetry. We study here only a linear regime in which the solution of our system of equations remains at all times close to a cylindrically symmetrical solution; in a realistic situation the deviations from this solution are likely larger and the streamer will sooner deviate significantly from the symmetrical solution. 


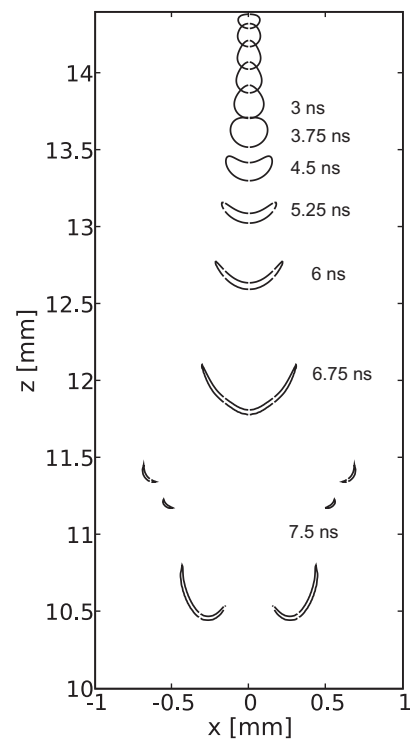

Fig. 3. Evolution of an axi-symmetrical streamer head represented by contours delimiting the region where charge density is larger than half of its maximum. The contours are plotted at regular time steps of $0.75 \mathrm{~ns}$. Note that the equivalent figure for the evolution of a streamer with a slight perturbation of the initial symmetry looks almost identical to this one due to the smallness of the perturbation.

In our simulation a negative streamer propagates in nitrogen (i.e. there is no photo-ionization nor electron losses via attachment) between parallel plates in a $14.4 \mathrm{~mm}$ gap with a homogeneous field of $50 \mathrm{kV} / \mathrm{cm}$. For the spatial discretization we use the nested-grids approach of Montijn et al. [22], discussed above. The highest resolution in $r$ and $z$ is $\Delta r=\Delta z=2.5 \mu \mathrm{m}$. The radial extension of the simulated domain was $4 \mathrm{~mm}$. For the Poisson equation, we used homogeneous Dirichlet boundary conditions at the upper and lower electrodes and homogeneous Neumann conditions at the radial boundary. In the solution of the reaction-drift-diffusion equation we used homogeneous Neumann boundary conditions in all boundaries.

First we run a simulation with perfectly symmetrical initial conditions consisting in a semispherical Gaussian neutral ionization seed attached to the upper electrode. To save time, this simulation was performed in a purely $2 \mathrm{D}$ code in a radial and a longitudinal coordinate $(r, z){ }^{2}$ The radius of the seed is $73.6 \mu \mathrm{m}$ and its highest electron and ion density is $2.6 \cdot 10^{10} \mathrm{~cm}^{-3}$. As seen in Fig. 3, the streamer branches between 6.75 and 7.5 ns. In this context, "branching" means that the streamer front loses its convexity even though in this case it remains cylindrically symmetrical; this is the branching studied in [76,77].

To show that this branching is indeed associated with the breaking of cylindrical symmetry observed in experiments, we run a similar simulation where the initial condition deviates slightly from cylindrical symmetry. In this simulation we used cylindrical coordinates with angular resolution $\Delta \theta=2 \pi / N, N=32$.

The asymmetrical initial state is constructed from the symmetrical initial electron densities $\bar{n}_{e}(r, z)$ by drawing $\eta_{1}, \ldots$, $\eta_{N-1}$ real numbers from a normal distribution $\mathcal{N}(0,1)$ and then setting

$$
\begin{aligned}
& \widetilde{n}_{e} 0(r, z, t=0)=\overline{n_{e}}(r, z), \\
& \tilde{n}_{e} k(r, z, t=0)=\epsilon\left(\eta_{k}+i \eta_{N-k}\right) \overline{n_{e}}(r, z) \frac{r}{R_{0}} \quad(0<k<N / 2) \text {, } \\
& \widetilde{n} e_{N / 2}(r, z, t=0)=\epsilon \eta_{N / 2} \overline{n_{e}}(r, z) \frac{r}{R_{0}}, \\
& \widetilde{n}_{e} k(r, z, t=0)=\tilde{n}_{e N-k}^{\star}(r, z, t=0) \quad(N / 2<k<N),
\end{aligned}
$$

where $\widetilde{n}_{e} k(r, z, t)$ is the discrete Fourier transform of the asymmetrical electron density, defined as in (6):

$$
\widetilde{n}_{e} k(r, z)=\sum_{n=0}^{N-1} n_{e}(r, z, 2 \pi n / N) e^{-2 \pi i k n / N} .
$$

The different cases in (12) arise because we have to impose $\widetilde{n}_{e} k=\widetilde{n}_{e} N-k^{\star}$, since $n_{e}$ is real. Note that we draw $N-1$ real numbers $\eta_{k}$ for the $N$ real numbers (including real and imaginary components) of the Fourier transform of a real quantity, with the $k=0$ mode remaining unperturbed.

\footnotetext{
${ }^{2}$ To rule out possible bugs in the code, we have checked that this 2D code and the 3D code with perfectly symmetrical initial conditions (i.e. restricted to the $k=0$ mode) give the same results within machine accuracy.
} 
The strength of the perturbation to the symmetrical state is $\epsilon$ and the factor $r / R_{0}$ is introduced to keep $n_{e}(r, z, \theta)$ continuous at $r=0$. The typical length $R_{0}$ is introduced to keep $\epsilon$ dimensionless. Here we take $R_{0}=2.3 \mu \mathrm{m}$ (the inverse of the Townsend multiplication rate), $\epsilon=10^{-8}$ but we also used $\epsilon=10^{-6}$ to check that we were indeed in a linear regime and the growth rates of the perturbation do not depend on $\epsilon$. We chose to perturb only the initial electron density, keeping the initial ion density symmetrical.

Note that this way of perturbing the electron density does not guarantee that it will remain positive. However, here we are interested in very small perturbations; in this limit the resulting density will almost certainly be positive everywhere and we checked for that.

To track the small deviations from perfect symmetry observed in the asymmetrical simulation we define

$$
W_{k}^{(u)}=\Delta \theta \int_{-\infty}^{+\infty} d z \int_{0}^{\infty} d r r \tilde{u}_{k}(r, z),
$$

where $u$ stands for either the electron density $n_{e}$ or the charge density $n_{i}-n_{e}$. The $W_{k}^{(u)}$ give us an idea of the "spectral content" of electrons or charges in each mode. Note that $W_{0}^{\left(n_{e}\right)}$ is the total number of electrons and $W_{0}^{\left(n_{i}-n_{e}\right)}$ is the total charge contained in the simulated volume. In general the $W_{k}^{(u)}$ are $N$ complex numbers $k=0, \ldots, N-1$ but since they come from the DFT of a real quantity, they satisfy relations analogous to (7); for $N$ even they reduce to $(N-2) / 2$ complex numbers and 2 reals. To analyze the spectral content of the electron density we look at the amplitudes $\left|W_{k}^{(u)}\right|=$ $\sqrt{W_{k}^{(u)} W_{k}^{(u) \star}}=\sqrt{W_{k}^{(u)} W_{N-k}^{(u)}}$ for $k=0, \ldots, N / 2$.

Still, plotting $\left|W_{k}^{(u)}(t)\right|$ is usually not the best way to visualize the evolution of the streamer. First, the number of electrons and the total charge changes in time; one should compare each mode with the corresponding total, $\left|W_{0}^{(u)}\right|$. Second, because we give random initial values to each $\left|W_{k}^{(u)}\right|$, it is also better to normalize them according to their initial value. Hence we define

$$
V_{k}^{(u)}(t)=\frac{\left|W_{k}^{(u)}(t)\right|\left|W_{0}^{(u)}(0)\right|}{\left|W_{0}^{(u)}(t)\right|\left|W_{k}^{(u)}(0)\right|},
$$

which satisfies $V_{0}^{(u)}(t)=1$ for all times, and $V_{k}^{(u)}(0)=1$ for all $k$. The evolution of the $V_{k}^{(u)}(t)$ is shown in Fig. 4 .

The evolution of the axial perturbation clearly shows a change of regime at $t=t_{B}$ with $t_{B} \approx 7$ ns, coinciding with the onset of streamer branching. For $t<t_{B}$ the evolution of the Fourier modes is smooth and all of them decay with respect to the $k=0$ mode $\left(d V_{k}^{\left(n_{e}\right)} / d t<0\right)$; during this phase the streamer is becoming more symmetrical. On the other hand for $t>t_{B}$ some modes $k \geqslant 1$ grow with respect to the $k=0$ mode. This is also shown in Fig. 5, where we represented the growth rates $\gamma$ of the unnormalized components $W_{k}^{\left(n_{i}-n_{e}\right)}$ of the space charge density; for each $k, \gamma$ is obtained by fitting $W_{k}^{(u)}(t)$ to an exponential $A e^{\gamma t}$ for $t<t_{B}$ and $t>t_{B}$. Also shown in Fig. 5 are the initial values of $\left|W_{k}^{\left(n_{i}-n_{e}\right)}\right|$; the plot does not show any clear correlation between these initial values and the subsequent growth rates.

Since up to $t_{B}$ the evolution of the perturbation is linear, the normalization of $V_{k}^{(u)}(t)$ assures that the values in Figs. 4 and 5 for $t<t_{B}$ are largely independent of the realization of the random variables $\eta_{1}, \ldots, \eta_{N-1}$ and of the overall magnitude of the perturbation $(\epsilon)$ as long as it remains small. The evolution after $t_{B}$ appears to couple different modes and depends significantly on the initial random perturbation.
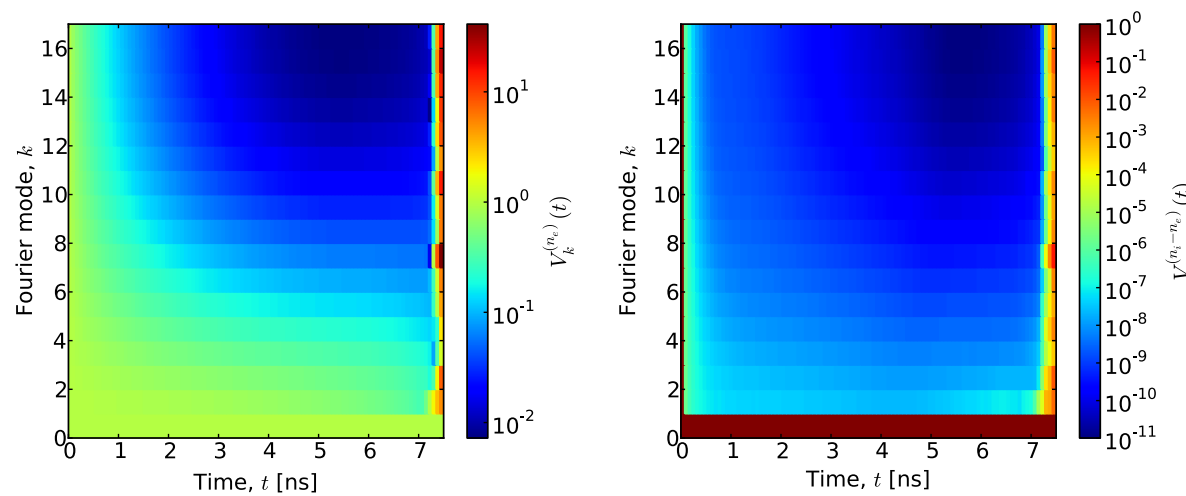

Fig. 4. Time dependence of the normalized Fourier components of the electron density $\left[V_{k}^{\left(n_{e}\right)}(t)\right.$; see text for a definition] (left) and the space charge density $\left[V_{k}^{\left(n_{i}-n_{e}\right)}(t)\right]$ (right) in a simulation with a slightly asymmetrical initial condition. Note that the $V_{k}^{(u)}(t)$ with $k>0$ decay smoothly up to $t=t_{B} \approx 7$ ns. This means that the $k=0$ component of the electron density (i.e. the total number of electrons) is growing faster than any other Fourier component; we interpret this as the streamer becoming closer to cylindrical symmetry. At $t=t_{B}$ a change in behavior is clear; after this points several $V_{k}^{(u)}(t)$ with $k>0$ grow faster than the $k=0$ mode and the cylindrical symmetry is gradually broken. 


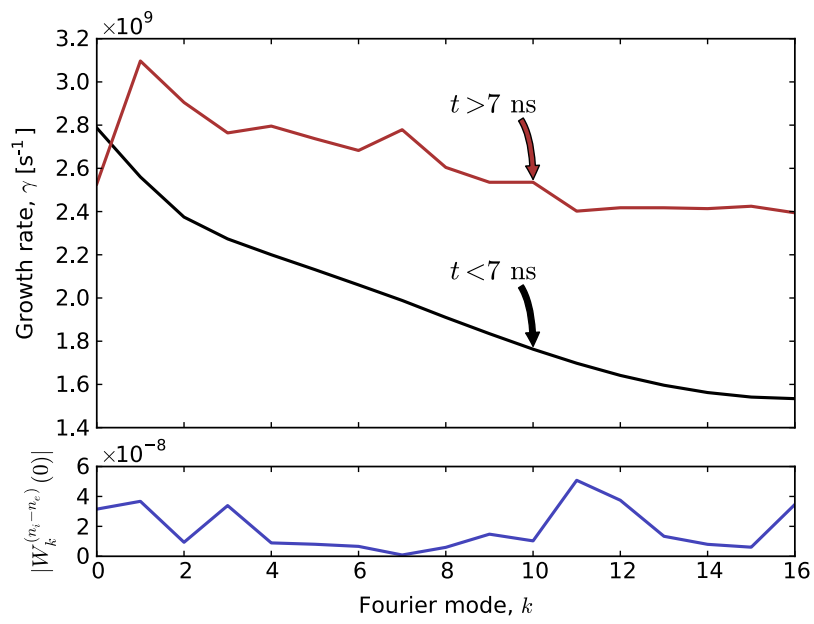

Fig. 5. Upper panel: Growth rate of the unnormalized Fourier components of the space charge density $\left[W_{k}^{\left(n_{i}-n_{e}\right)}\right]$. We plot separately the growth rates before and after the branching time $t_{B} \approx 7$ ns. Note that for $t>t_{B}$ several Fourier modes grow faster than the $k_{0}$ mode, indicating that the shape of the streamer deviates from cylindrical symmetry. Lower panel: Initial perturbation to $\left|W_{k}^{\left(n_{i}-n_{e}\right)}\right|$. It does not appear to be any clear correlation between the initial perturbation and the growth rate of each Fourier mode.

These results show that the "branching" found in a simulation with cylindrical symmetry as shown in Fig. 3, i.e. as nonconvexity of an axi-symmetric solution translates, in a full 3D simulation, into a faster growth of the axially non-symmetric modes relative to the $k=0$ mode and hence into "branching" in the more conventional sense of bifurcation of a single channel.

We note that there are two possible reasons for this outcome: (a) that the streamer reaches a state of general dynamical instability that simultaneously affects both the cylindrically symmetrical and the asymmetrical modes and (b) that a nonconvex, cylindrically symmetric streamer head is unstable against perturbations of its symmetry; this is expected if the electric field has a significant off-axis peak. These two processes are compatible and likely both play a role.

\subsection{Streamers in inhomogeneous media: sprite streamers}

Up to now, we have only considered streamers propagating in homogeneous media. Streamers models have been extended to inhomogeneous gas densities and compositions in order to study the interaction between a streamer and gas bubbles [78] and the propagation of a streamer inside a plasma jet [79]. Here we consider another important case where streamers propagate in an inhomogeneous gas, namely sprites [80]. These are transient discharges in the upper atmosphere above active thunderclouds, where air density varies with altitude. Sprites span altitudes from about $50 \mathrm{~km}$ up to $90 \mathrm{~km}$, are tens of kilometers wide and usually last for some milliseconds. Often they are composed of two separate regions: an upper, diffuse one usually extending from about 80 to $90 \mathrm{~km}$ altitude and a lower, filamentary region composed by hundreds of streamers [81,19] (sometimes called "sprite tendrils"). Pasko has recently reviewed models of single sprite streamers propagating in homogeneous air density [82].

A single streamer in the filamentary region can extend for tens of kilometers in altitude. In this range the density of air varies considerably (roughly by a factor of 2 every $5 \mathrm{~km}$ ). Typical streamer lengths approximately scale with density [18] so the density variation introduces another length scale on top of those discussed above. The first numerical models to include the variation of air density in sprite streamers were published recently [83,84].

\subsubsection{Numerical implementation}

In principle, a dependence on altitude can be incorporated easily into streamer models. Since the air density $n$ is a known function of altitude, the effect of a gradient of density can be incorporated in altitude-dependent transport parameters $\left(\mu_{e}\right.$ and $D_{e}$ in (1a)) and reaction rates.

The calculation of the photo-ionization term is more complex. At lower altitudes, photons are absorbed faster and propagate over shorter distances; the absorption becomes dependent on the photon propagation path and photo-ionization is not isotropic. The work-around proposed in [83] is to assume that (a) the emission of photo-ionizing radiation is very localized around a streamer head and (b) the absorption length of photo-ionization is always much shorter than the scale of variation of the atmospheric density (about $7.2 \mathrm{~km}$ ). In that case one can use an isotropic absorption with absorption lengths accurate only close to the streamer head. This is implemented by finding, at each time, the location of the maximum of $S^{i m}$ along the streamer axis and using its altitude to obtain the parameters of the function $h$ in (2) which is then applied in the complete domain. 


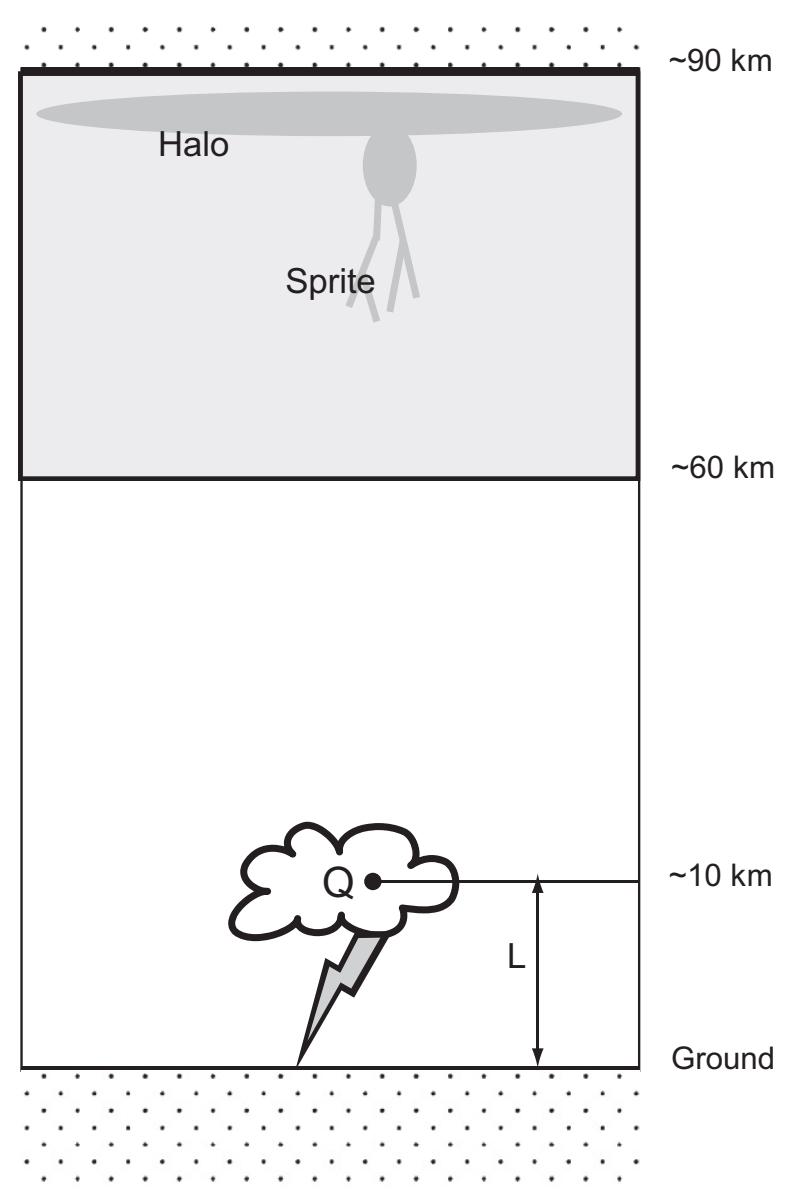

Fig. 6. Scheme of the geometry of sprite streamer simulations. First the field of the cloud charge with boundary conditions on ground and ionosphere is solved analytically. The Poisson equation for the sprite charges is solved in the complete domain between ground and ionosphere; the reaction-driftdiffusion equations are solved only in the upper, shaded volume.

Finally, we note that in a scheme with adaptive refinement based on the electric field, the criterium (5) must be updated to refer not to the electric field but to the reduced electric field $E / n$, where $n$ is gas density, which is the relevant quantity when the air density is not homogeneous.

\subsubsection{Discharge geometry}

The geometry of sprite simulations is depicted schematically in Fig. 6 . The electrical field is created when a thunder stroke at an altitude $L \approx 10 \mathrm{~km}$ moves a large amount of charge to the ground. Most sprites are generated after positive cloud-toground lightning strokes; these strokes leave a negative charge $Q$ in the cloud. In the quasi-electrostatic approximation [85] $Q$ is taken to change so slowly in time that the electromagnetic radiative terms can be neglected (i.e. the speed of light is infinite). The upper and lower boundaries of the resulting electrostatic problem are, respectively, the Earth's surface and a horizontal layer in the ionosphere at about $85-90 \mathrm{~km}$ altitude, where the Maxwell relaxation time due to the atmospheric conductivity is much shorter than the characteristic times involved in the discharge.

In principle, the charge $Q$ can be included in the simulation domain and treated as a prescribed source term in the Poisson equation. However, the distance between this charge and the upper boundary of the simulation is about $80 \mathrm{~km}$ and in order to minimize the effects of the artificial lateral boundaries the domain would have to be extended to a radius much larger than these $80 \mathrm{~km}$. To avoid this overextension, in [83] the field created by $Q$ was calculated semi-analytically as the field created by a charge at a distance $L$ from the lower of two parallel electrodes. This is expressed as a sum of the fields created by an infinite number of image charges; this sum is truncated after 4 terms ( 2 on each side). To this one adds the self-consistent field of the sprite, calculated by solving the Poisson equation with homogeneous Dirichlet $(\phi=0)$ boundary conditions in the upper and lower electrodes. The horizontal width of the domain must be larger than the lateral extension of the space charges to minimize the effects of artificial boundaries but it can still be smaller that the distance between $Q$ and the sprite.

Once the field is calculated, the reaction-drift-diffusion equations must only be solved in a smaller volume (see Fig. 6). In [83] the volume was a cylinder from 55 to $85 \mathrm{~km}$ in altitude, with a radius of $20 \mathrm{~km}$. 

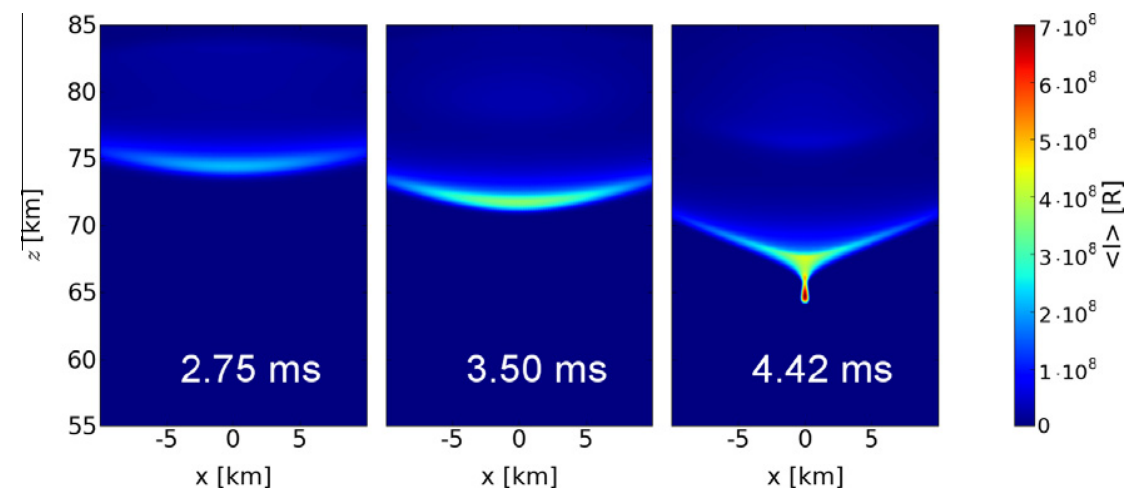

Fig. 7. Optical emissions emitted from a streamer emerging from the sharpened edge of a relaxation-ionization wave from the ionosphere. This wave produces diffuse optical emissions observed as a halo. Reproduced from [83].

\subsubsection{Results}

A notable difference between streamers in the laboratory and sprite streamers is that in a laboratory they are almost always initiated from a sharp metallic electrode that is clearly not present in the upper atmosphere. Raizer et al. [81] proposed that streamers emerge from "plasma patches" created by electro-magnetic waves [86]. Recently, Marshall et al. [87] used a finite difference, full electromagnetic code to evaluate the influence of lightning-generated electromagnetic pulses (EMP) on the electron density in the upper atmosphere, reporting large enhancements of up to a factor 4 for repeated EMP's.

However, an isolated patch would create a two-headed streamer propagating simultaneously up and down [29]. In observations, however, sprite streamers always start propagating downwards [88,89]. In [83] it was shown how a single, downwards-propagating sprite streamer emerges from the destabilization of a sharp screening-ionization wave from the ionosphere; Fig. 7 shows the optical emissions resulting from that process. The initial condition amounted here to air density exponentially decreasing with altitude and electron density increasing with altitude as in fair weather night-time.

A relevant recent result related to sprite streamers concerns the trailing optical emissions emanating from the streamer body at a certain distance from the head. Although initially this luminosity was attributed to chemical processes in the wake of the streamer head [90,91], two recent papers [92,84] used streamer density models to show that it originates from a re-enhancement of the electric field. This occurs if there is a substantial electrical current in the streamer body, generated because the net charge in the streamer head increases during propagation. In [84] the electric field in this re-enhancement approached $E \approx E_{k}$; this appears to be dynamically stable in a current-carrying state.

The most important challenges in the simulation of sprites with density models are the simulation of long propagation distances of tens of kilometers and the effects of streamer interaction. As we commented in Section 4.1, the development of reduced models appears highly desirable. An additional difficulty in the modeling of sprite discharges is our imperfect knowledge of the dynamics of the mesosphere. For example, the spatial distribution of free electrons before a sprite is initiated is at present very uncertain.

\section{Outlook}

As with any scientific endeavor, it is hard to predict the future of streamer density models. The ultimate aim of simulating and understanding a fully branched tree of streamers strictly within the model described in this paper looks unrealistic in the short and medium term.

On the other hand, gradual improvements to density models will surely come in the short run. There are two obvious directions to extend the physical models. In the "microscopic direction" the modeling of the microscopic phenomena is improved; this includes e.g. a more accurate calculation of the photo-ionization sources, additional chemical processes and the inclusion of stochasticity, converging towards detailed particle and hybrid models. The "macroscopic direction," extends the simulation domain, possibly to include many interacting streamers and also incorporate in an improved fashion the interaction between the streamer and its surroundings. Although in principle they are compatible, both directions demand a higher computational effort, and it is unlikely that they will be combined in a practicable unified model. Even if approached separately, they will likely require faster computers and numerical algorithms.

However, it may not be necessary to describe the full range of physics, from the microscopic to the macroscopic level, inside a single model. A more promising approach is to develop a hierarchy of models of decreasing microscopic detail. Each model would rely on simplifications and assumptions extracted from models down in the hierarchy or from direct experimental observations. Density models play a pivotal role in this hierarchy, sitting between particle models and higher-level descriptions of a streamer tree such as the dielectric breakdown model of Niemeyer et al. [93] Pasko et al. [94], phenomenological branching models [95] and moving boundary models [6-10,23]. 


\section{Acknowledgements}

We thank C. Li for useful comments on this paper. AL was supported by the Spanish Ministry of Science and Innovation, MICINN under project AYA2009-14027-C05-02 and by the Junta de Andalucia, Proyecto de Excelencia FQM-5965.

\section{References}

[1] H. Raether, Die Entwicklung der Elektronenlawine in den Funkenkanal, Zeitschrift fur Physik 112 (1939) 464, doi:10.1007/BF01340229.

[2] Y.P. Raizer, Gas Discharge Physics, Springer Verlag, Berlin, Germany, 1991.

[3] U. Ebert, C. Montijn, T.M.P. Briels, W. Hundsdorfer, B. Meulenbroek, A. Rocco, E.M. van Veldhuizen, The multiscale nature of streamers, Plasma Sour. Sci. Technol. 15 (2006) S118, doi:10.1088/0963-0252/15/2/S14. <arXiv:physics/0604023>.

[4] U. Ebert, D.D. Sentman, Streamers, sprites, leaders, lightning: from micro- to macroscales, J. Phys. D 41 (23) (2008), doi:10.1088/0022-3727/41/23/ 230301. <arXiv:0811.2075>.

[5] C. Li, U. Ebert, W. Hundsdorfer, Spatially hybrid computations for streamer discharges: II. fully 3D computations, J. Comput. Phys., this issue.

[6] E.D. Lozansky, O.B. Firsov, Theory of the initial stage of streamer propagation, J. Phys. D 6 (1973) 976, doi:10.1088/0022-3727/6/8/310.

[7] B. Meulenbroek, A. Rocco, U. Ebert, Streamer branching rationalized by conformal mapping techniques, Phys. Rev. E 69 (6) (2004) 9067402, doi:10.1103/PhysRevE.69.067402. <arXiv:physics/0305112>.

[8] F. Brau, A. Luque, B. Meulenbroek, U. Ebert, L. Schäfer, Construction and test of a moving boundary model for negative streamer discharges, Phys. Rev. E 77 (2) (2008) 026219, doi:10.1103/PhysRevE.77.026219. <arXiv:0707.1402>.

[9] F. Brau, B. Davidovitch, U. Ebert, Moving boundary approximation for curved streamer ionization fronts: solvability analysis, Phys. Rev. E 78 (5) (2008) 056212, doi:10.1103/PhysRevE.78.056212. <arXiv:0807.4614>.

[10] F. Brau, A. Luque, B. Davidovitch, U. Ebert, Moving-boundary approximation for curved streamer ionization fronts: numerical tests, Phys. Rev. E 79 (6) (2009) 066211, doi:10.1103/PhysRevE.79.066211. 〈arXiv:0901.1916>.

[11] C. Li, U. Ebert, W. Hundsdorfer, Spatially hybrid computations for streamer discharges with generic features of pulled fronts: I. Planar fronts, J. Comput. Phys. 229 (2010) 200, doi:10.1016/j.jcp.2009.09.027. <arXiv:0904.2968>.

[12] C. Li, W.J.M. Brok, U. Ebert, J.J.A.M. van der Mullen, Deviations from the local field approximation in negative streamer heads, J. Appl. Phys. 101 (12) (2007) 123305, doi:10.1063/1.2748673. <arXiv:physics/0702129>.

[13] C. Li, U. Ebert, W. Hundsdorfer, 3D hybrid computations for streamer discharges and production of runaway electrons, J. Phys. D 42 (20) (2009) 202003, doi:10.1088/0022-3727/42/20/202003. <arXiv:0907.0555>.

[14] O. Chanrion, T. Neubert, Production of runaway electrons by negative streamer discharges, J. Geophys. Res. (Space Phys) 115 (2010) A00E32, doi:10.1029/2009JA014774.

[15] A.V. Phelps, B.M. Jelenkovich, L.C. Pitchford, Simplified models of electron excitation and ionization at very high E/n, Phys. Rev. A 36 (1987) 5327, doi:10.1103/PhysRevA.36.5327.

[16] G.D. Moss, V.P. Pasko, N. Liu, G. Veronis, Monte Carlo model for analysis of thermal runaway electrons in streamer tips in transient luminous events and streamer zones of lightning leaders, J. Geophys. Res. (Space Phys) 111 (2006) A02307, doi:10.1029/2005JA011350.

[17] S.B. Vrhovac, V.D. Stojanović, B.M. Jelenković, Z.L. Petrović, Energy distributions of electrons in a low-current self-sustained nitrogen discharge, J. Appl. Phys. 90 (2001) 5871, doi:10.1063/1.1415364.

[18] U. Ebert, S. Nijdam, C. Li, A. Luque, T. Briels, E. van Veldhuizen, Review of recent results on streamer discharges and discussion of their relevance for sprites and lightning, J. Geophys. Res. (Space Phys) 115 (2010) A00E43, doi:10.1029/2009JA014867. <arXiv:1002.0070>.

[19] V.P. Pasko, U.S. Inan, T.F. Bell, Spatial structure of sprites, Geophys. Res. Lett. 25 (1998) 2123, doi:10.1029/98GL01242.

[20] G. Wormeester, S. Pancheshnyi, A. Luque, S. Nijdam, U. Ebert, Probing photo-ionization: simulations of positive streamers in varying $\mathrm{N}_{2}: \mathrm{O}_{2}$-mixtures, J. Phys. D 43 (2010) 505201, doi:10.1088/0022-3727/43/50/505201. <arXiv:1008.3309>

[21] S. Nijdam, F.M.J.H. van de Wetering, R. Blanc, E.M. van Veldhuizen, U. Ebert, Probing photo-ionization: experiments on positive streamers in pure gases and mixtures, J. Phys. D 43 (14) (2010) 145204, doi:10.1088/0022-3727/43/14/145204. <arXiv:0912.0894>.

[22] C. Montijn, W. Hundsdorfer, U. Ebert, An adaptive grid refinement strategy for the simulation of negative streamers, J. Comput. Phys. 219 (2006) 801 , doi:10.1016/j.jcp.2006.04.017. <arXiv:physics/0603070>.

[23] U. Ebert, F. Brau, G. Derks, W. Hundsdorfer, C. Kao, C. Li, A. Luque, B. Meulenbroek, S. Nijdam, V. Ratushnaya, L. Schäfer, S. Tanveer, Multiple scales in streamer discharges, with an emphasis on moving boundary approximations, Nonlinearity 24 (2011) C1, doi:10.1088/0951-7715/24/1/C01.

[24] O. Chanrion, T. Neubert, A PIC-MCC code for simulation of streamer propagation in air, J. Comput. Phys. 227 (2008) 7222, doi:10.1016/ j.jcp.2008.04.016.

[25] C. Soria-Hoyo, F. Pontiga, A. Castellanos, based procedure for the integration of multiple time scale problems in gas discharge physics, J. Comput. Phys. 228 (2009) 1017, doi:10.1016/j.jcp.2008.10.007.

[26] S. Pancheshny, Role of electronegative gas admixtures in streamer start propagation and branching phenomena, Plasma Sour. Sci. Technol. 14 (2005) 645, doi:10.1088/0963-0252/14/4/002.

[27] M.B. Zhelezniak, A.K. Mnatsakanian, S.V. Sizykh, Photoionization of nitrogen and oxygen mixtures by radiation from a gas discharge, Teplofizika Vysokikh Temperatur 20 (1982) 423.

[28] W. Legler, Anregung von UV-Strahlung in Stickstoff und Wasserstoff durch einen Elektronenschwarm, Z. Phys. (1963) 169-183.

[29] N. Liu, V.P. Pasko, Effects of photoionization on propagation and branching of positive and negative streamers in sprites, J. Geophys. Res. (Space Phys) 109 (2004) A04301, doi:10.1029/2003JA010064.

[30] G.V. Naidis, On photoionization produced by discharges in air, Plasma Sour. Sci. Technol. 15 (2006) 253, doi:10.1088/0963-0252/15/2/010.

[31] A.A. Kulikovsky, The role of photoionization in positive streamer dynamics, J. Phys. D 33 (2000) 1514, doi:10.1088/0022-3727/33/12/314.

[32] D. Dubrovin, S. Nijdam, E.M. van Veldhuizen, U. Ebert, Y. Yair, C. Price, Sprite discharges on Venus and Jupiter-like planets: a laboratory investigation, J. Geophys. Res. (Space Phys) 115 (2010) A00E34, doi:10.1029/2009JA014851. <arXiv:1003.0207>.

[33] A.V. Phelps, L.C. Pitchford, Anisotropic scattering of electrons by N2 and its effect on electron transport, Phys. Rev. A 31 (1985) 2932, doi:10.1103/ PhysRevA.31.2932.

[34] G.J.M. Hagelaar, L.C. Pitchford, Solving the Boltzmann equation to obtain electron transport coefficients and rate coefficients for fluid models, Plasma Sour. Sci. Technol. 14 (2005) 722, doi:10.1088/0963-0252/14/4/011.

[35] S. Dujko, U. Ebert, R. White, Z.L. Petrovic, Electron transport data in $\mathrm{N}_{2}-\mathrm{O}_{2}$ streamer plasma discharges, Publ. Astron. Obs. Belgrade 89 (2010) $71-74$.

[36] S.K. Dhali, P.F. Williams, Numerical simulation of streamer propagation in nitrogen at atmospheric pressure, Phys. Rev. A 31 (1985) 1219, doi:10.1103/ PhysRevA.31.1219.

[37] S.K. Dhali, P.F. Williams, Two-dimensional studies of streamers in gases, J. Appl. Phys. 62 (1987) 4696, doi:10.1063/1.339020.

[38] C. Wu, E.E. Kunhardt, Formation and propagation of streamers in $\mathrm{N}_{2}$ and $\mathrm{N}_{2}-\mathrm{SF}_{6}$ mixtures, Phys. Rev. A 37 (1988) 4396, doi:10.1103/PhysRevA.37.4396

[39] P.A. Vitello, B.M. Penetrante, J.N. Bardsley, Simulation of negative-streamer dynamics in nitrogen, Phys. Rev. E 49 (1994) 5574, doi:10.1103/ PhysRevE.49.5574.

[40] S.T. Zalesak, Fully multidimensional flux-corrected transport algorithms for fluids, J. Comput. Phys. 31 (1979) 335, doi:10.1016/0021-999(79)90051-2.

[41] A. Kulikovsky, A more accurate Scharfetter-Gummel algorithm of electron transport for semiconductor and gas discharge simulation, J. Comput. Phys. 119 (1995) 149, doi:10.1006/jcph.1995.1123. 
[42] A. Bourdon, V.P. Pasko, N.Y. Liu, S. Célestin, P. Ségur, E. Marode, Efficient models for photoionization produced by non-thermal gas discharges in air based on radiative transfer and the Helmholtz equations, Plasma Sour. Sci. Technol. 16 (2007) 656, doi:10.1088/0963-0252/16/3/026.

[43] S.V. Pancheshnyi, A.Y. Starikovskii, Two-dimensional numerical modelling of the cathode-directed streamer development in a long gap at high voltage, J. Phys. D 36 (2003) 2683, doi:10.1088/0022-3727/36/21/014.

[44] B. Koren, A robust upwind discretization method for advection, diffusion and source terms, in: C.B. Vreugdenhil, B. Koren, (Eds.), Numerical Methods for Advection-Diffusion Problems, Vieweg Verlag, Wiesbaden, 1993, pp. 117-137.

[45] S. Pancheshnyi, M. Nudnova, A. Starikovskii, Development of a cathode-directed streamer discharge in air at different pressures: experiment and comparison with direct numerical simulation, Phys. Rev. E 71 (1) (2005) 016407, doi:10.1103/PhysRevE.71.016407.

[46] D. Bessières, J. Paillol, A. Bourdon, P. Ségur, E. Marode, A new one-dimensional moving mesh method applied to the simulation of streamer discharges, J. Phys. D 40 (2007) 6559, doi:10.1088/0022-3727/40/21/016

[47] O. Eichwald, O. Ducasse, D. Dubois, A. Abahazem, N. Merbahi, M. Benhenni, M. Yousfi, Experimental analysis and modelling of positive streamer in air: towards an estimation of $O$ and N radical production, J. Phys. D 41 (23) (2008) 234002, doi:10.1088/0022-3727/41/23/234002.

[48] W. Min, H. Kim, S. Lee, S. Hahn, A study on the streamer simulation using adaptive mesh generation and FEM-FCT, IEEE Trans. Magn. 37 (2001) 3141 , doi:10.1109/20.952562.

[49] D.S. Nikandrov, R.R. Arslanbekov, V.I. Kolobov, Streamer simulations with dynamically adaptive Cartesian mesh, IEEE Trans. Plasma Sci. 36 (2008) 932 , doi:10.1109/TPS.2008.924533.

[50] S. Pancheshnyi, P. Ségur, J. Capeillère, A. Bourdon, Numerical simulation of filamentary discharges with parallel adaptive mesh refinement, J. Comput. Phys. 227 (2008) 6574, doi:10.1016/j.jcp.2008.03.020.

[51] U. Ebert, W. van Saarloos, Front propagation into unstable states: universal algebraic convergence towards uniformly translating pulled fronts, Phys. D Nonlinear Phenom. 146 (2000) 1, doi:10.1016/S0167-2789(00)00068-3. <arXiv:cond-mat/0003181>.

[52] A.A. Kulikovsky, Positive streamer between parallel plate electrodes in atmospheric pressure air, J. Phys. D 30 (1997) 441, doi:10.1088/0022-3727/30/ $3 / 017$.

[53] S.V. Pancheshnyi, S.M. Starikovskaia, A.Y. Starikovskii, Role of photoionization processes in propagation of cathode-directed streamer, J. Phys. D 34 (2001) 105, doi:10.1088/0022-3727/34/1/317.

[54] J. Wackers, A nested-grid direct Poisson solver for concentrated source terms, J. Comput. Appl. Math. 180 (2005) 1, doi:10.1016/j.cam.2004.09.054.

[55] S. Celestin, Z. Bonaventura, B. Zeghondy, A. Bourdon, P. Ségur, The use of the ghost fluid method for Poisson's equation to simulate streamer propagation in point-to-plane and point-to-point geometries, J. Phys. D 42 (6) (2009) 0652030, doi:10.1088/0022-3727/42/6/065203.

[56] A.A. Kulikovsky, Three-dimensional simulation of a positive streamer in air near curved anode, Phys. Lett. A 245 (1998) 445, doi:10.1016/S0375960(98)00415-0.

[57] A. Luque, U. Ebert, W. Hundsdorfer, Interaction of streamer discharges in air and other oxygen-nitrogen mixtures, Phys. Rev. Lett. 101 (7) (2008) 075005, doi:10.1103/PhysRevLett.101.075005. <arXiv:0712.2774>.

[58] N.Y. Babaeva, G.V. Naidis, Two-dimensional modelling of positive streamer dynamics in non-uniform electric fields in air, J. Phys. D 29 (1996) 2423 , doi:10.1088/0022-3727/29/9/029.

[59] A. Luque, V. Ratushnaya, U. Ebert, Positive and negative streamers in ambient air: modelling evolution and velocities, J. Phys. D 41 (23) (2008) 234005, doi:10.1088/0022-3727/41/23/234005. <arXiv:0804.3539>.

[60] H. Singer, H. Steinbigler, P. Weiss, A charge simulation method for the calculation of high voltage fields, IEEE Trans. Power Appar. Syst. PAS-93 (5) (1974) 1660-1668, doi:10.1109/TPAS.1974.293898.

[61] N. Malik, A review of the charge simulation method and its applications, IEEE Trans. Electr. Insul. 24 (1989) 3-20.

[62] C. Li, Joining particle and fluid aspects in streamer simulations, Ph.D. Thesis, Technische Universiteit Eindhoven, 2009. <http://repository.tue.nl/ 640104>.

[63] Y.V. Serdyuk, A. Larsson, S.M. Gubanski, M. Akyuz, The propagation of positive streamers in a weak and uniform background electric field, J. Phys. D 34 (2001) 614, doi:10.1088/0022-3727/34/4/323.

[64] J. van Dijk, K. Peerenboom, M. Jimenez, D. Mihailova, J. van der Mullen, The plasma modelling toolkit Plasimo, J. Phys. D 42 (19) (2009) 194012, doi:10.1088/0022-3727/42/19/194012.

[65] G. Steinle, D. Neundorf, W. Hiller, M. Pietralla, Two-dimensional simulation of filaments in barrier discharges, J. Phys. D 32 (1999) 1350, doi:10.1088/ $0022-3727 / 32 / 12 / 311$.

[66] P. Ségur, A. Bourdon, E. Marode, D. Bessieres, J.H. Paillol, The use of an improved Eddington approximation to facilitate the calculation of photoionization in streamer discharges, Plasma Sour. Sci. Technol. 15 (2006) 648, doi:10.1088/0963-0252/15/4/009.

[67] A. Luque, U. Ebert, C. Montijn, W. Hundsdorfer, Photoionization in negative streamers: Fast computations and two propagation modes, Appl. Phys. Lett. 90 (8) (2007) 081501, doi:10.1063/1.2435934. <arXiv:physics/0609247>.

[68] N. Liu, S. Célestin, A. Bourdon, V.P. Pasko, P. Ségur, E. Marode, Application of photoionization models based on radiative transfer and the Helmholtz equations to studies of streamers in weak electric fields, Appl. Phys. Lett. 91 (21) (2007) 211501, doi:10.1063/1.2816906.

[69] J. Capeillère, P. Ségur, A. Bzourdon, S. Célestin, S. Pancheshnyi, The finite volume method solution of the radiative transfer equation for photon transport in non-thermal gas discharges: application to the calculation of photoionization in streamer discharges, J. Phys. D 41 (23) (2008) 234018, doi:10.1088/0022-3727/41/23/234018.

[70] G.V. Naidis, On streamer interaction in a pulsed positive corona discharge, J. Phys. D 29 (1996) 779, doi:10.1088/0022-3727/29/3/039.

[71] A. Luque, F. Brau, U. Ebert, Saffman-Taylor streamers: mutual finger interaction in spark formation, Phys. Rev. E 78 (1) (2008) 016206, doi:10.1103/ PhysRevE.78.016206. <arXiv:0708.1722>

[72] V. Ratushnaya, A. Luque, U. Ebert, Electrodynamic characterization of long positive streamers in air, in preparation.

[73] P.G. Saffman, G. Taylor, The penetration of a fluid into a porous medium or hele-shaw cell containing a more viscous liquid, R. Soc. London Proc. Ser. A 245 (1958) 312, doi:10.1098/rspa.1958.0085.

[74] D. Bensimon, L.P. Kadanoff, S. Liang, B.I. Shraiman, C. Tang, Viscous flows in two dimensions, Rev. Mod. Phys. 58 (1986) 977, doi:10.1103/ RevModPhys.58.977.

[75] B. Meulenbroek, U. Ebert, L. Schäfer, Regularization of moving boundaries in a Laplacian field by a mixed Dirichlet-Neumann boundary condition: exact results, Phys. Rev. Lett. 95 (19) (2005) 195004, doi:10.1103/PhysRevLett.95.195004. <arXiv:nlin/0507019>.

[76] M. Arrayás, U. Ebert, W. Hundsdorfer, Spontaneous branching of anode-directed streamers between planar electrodes, Phys. Rev. Lett. 88 (17) (2002) 174502, doi:10.1103/PhysRevLett.88.174502. <arXiv:nlin/0111043>.

[77] C. Montijn, U. Ebert, W. Hundsdorfer, Numerical convergence of the branching time of negative streamers, Phys. Rev. E 73 (6) (2006), doi:10.1103/ PhysRevE.73(065401). <arXiv:physics/0604012>.

[78] N.Y. Babaeva, M.J. Kushner, Streamer branching: the role of inhomogeneities and bubbles, IEEE Trans. Plasma Sci. 36 (2008) 892, doi:10.1109/ TPS.2008.922434.

[79] G.V. Naidis, Modelling of streamer propagation in atmospheric-pressure helium plasma jets, J. Phys. D 43 (2010) 2001, doi:10.1088/0022-3727/43/40/ 402001.

[80] R.C. Franz, R.J. Nemzek, J.R. Winckler, Television image of a large upward electrical discharge above a thunderstorm system, Science 249 (1990) 48 , doi:10.1126/science.249.4964.48.

[81] Y.P. Raizer, G.M. Milikh, M.N. Shneider, S.V. Novakovski, Long streamers in the upper atmosphere above thundercloud, J. Phys. D 31 (1998) 3255 , doi:10.1088/0022-3727/31/22/014

[82] V.P. Pasko, Recent advances in theory of transient luminous events, J. Geophys. Res. (Space Phys) 115 (2010) A00E35, doi:10.1029/2009JA014860. 
[83] A. Luque, U. Ebert, Emergence of sprite streamers from screening-ionization waves in the lower ionosphere, Nat. Geosci. 2 (2009) 757, doi:10.1038/ ngeo662.

[84] A. Luque, U. Ebert, Sprites in varying air density: charge conservation glowing negative trails and changing velocity, Geophys. Res. Lett. 37 (2010) L06806, doi:10.1029/2009GL041982.

[85] V.P. Pasko, U.S. Inan, T.F. Bell, Y.N. Taranenko, Sprites produced by quasi-electrostatic heating and ionization in the lower ionosphere, J. Geophys. Res. 102 (1997) 4529, doi:10.1029/96JA03528.

[86] J.A. Valdivia, G. Milikh, K. Papadopoulos, Red sprites: lightning as a fractal antenna, Geophys. Res. Lett. 24 (1997) 3169, doi:10.1029/97GL03188.

[87] R.A. Marshall, R.T. Newsome, N.G. Lehtinen, N. Lavassar, U.S. Inan, Optical signatures of radiation belt electron precipitation induced by ground-based VLF transmitters, J. Geophys. Res. (Space Phys) 115 (2010) A08206, doi:10.1029/2010JA015394.

[88] S.A. Cummer, N. Jaugey, J. Li, W.A. Lyons, T.E. Nelson, E.A. Gerken, Submillisecond imaging of sprite development and structure, Geophys. Res. Lett. 33 (2006) L04104, doi:10.1029/2005GL024969.

[89] H.C. Stenbaek-Nielsen, M.G. McHarg, High time-resolution sprite imaging: observations and implications, J. Phys. D 41 (23) (2008) 234009, doi:10.1088/0022-3727/41/23/234009.

[90] T. Kanmae, H.C. Stenbaek-Nielsen, M.G. McHarg, Altitude resolved sprite spectra with 3 ms temporal resolution, Geophys. Res. Lett. 34 (2007) L07810, doi:10.1029/2006GL028608.

[91] D.D. Sentman, H.C. Stenbaek-Nielsen, M.G. McHarg, J.S. Morrill, Correction to Plasma chemistry of sprite streamers, J. Geophys. Res. (Atmos.) 113 (2008) D14399, doi:10.1029/2008JD010634.

[92] N. Liu, Model of sprite luminous trail caused by increasing streamer current, Geophys. Res. Lett. 37 (2010) L04102, doi:10.1029/2009GL042214.

[93] L. Niemeyer, L. Pietronero, H.J. Wiesmann, Fractal dimension of dielectric breakdown, Phys. Rev. Lett. 52 (1984) 1033, doi:10.1103/ PhysRevLett.52.1033.

[94] V.P. Pasko, U.S. Inan, T.F. Bell, Fractal structure of sprites, Geophys. Res. Lett. 27 (2000) 497, doi:10.1029/1999GL010749.

[95] M. Akyuz, A. Larsson, V. Cooray, G. Strandberg, 3d simulations of streamer branching in air, J. Electrost. 59 (2) (2003) 115-141, doi:10.1016/S0304388(03)00066-4. 